\title{
A Recursive Approach to the Kauffman Bracket
}

\author{
Abdul Rauf Nizami, Mobeen Munir, Umer Saleem, Ansa Ramzan \\ Division of Science and Technology, University of Education, Lahore, Pakistan \\ Email: arnizami@ue.edu.pk, mobeenmunir@gmail.com, umerlinks@hotmail.com, ansaramzan@yahoo.com
}

Received 26 July 2014; revised 28 August 2014; accepted 10 September 2014

Copyright (C) 2014 by authors and Scientific Research Publishing Inc.

This work is licensed under the Creative Commons Attribution International License (CC BY). http://creativecommons.org/licenses/by/4.0/

c) (7) Open Access

\section{Abstract}

We introduce a simple recursive relation and give an explicit formula of the Kauffman bracket of two-strand braid link $\widehat{x_{1}^{n}}$. Then, we give general formulas of the bracket of the sequence of links of three-strand braids $\alpha(n)=x_{1} x_{2} x_{1} x_{2} \cdots$. Finally, we give an interesting result that the Kauffman bracket of the three-strand braid link $\widehat{x_{1}^{m} x_{2}^{n}}$ is actually the product of the brackets of the twostrand braid links $\widehat{x_{1}^{m}}$ and $\widehat{x_{1}^{n}}$. Moreover, a recursive relation for $\left\langle\widehat{x_{1}^{a} x_{2}^{b} x_{1}^{c} x_{2}^{d}}\right\rangle$ is also given.

\section{Keywords}

\section{Recursive Relation, Kauffman Bracket, Braid Link}

\section{Introduction}

The Kauffman bracket polynomial was introduced by L. H. Kauffman in 1987 [1] in concern with link invariants. The bracket polynomial soon became popular due to its connections with the Jones polynomial, dichromatic polynomial, and the Potts model. While the HOMPLY polynomial and the bracket polynomial are distinct with different topological properties, there is a very beautiful relationship between them due to F. Jaeger [2], and it is also observed in a special case by Reshetikhin [3].

The Kauffman bracket (polynomial) is actually not a link invariant because it is not invariant under the first Reidemeister move. However, it has many applications and it can be extended to a popular link invariant, the Jones polynomial. In the present work we shall confine ourselves to the Kauffman bracket to avoid this work from unnecessary length and to leave it for applications.

This paper is organized as follows: In Section 2 we shall give the basic ideas about knots, braids, and the Kauffman bracket. In Section 3 we shall present the main results. 


\section{Basic Notions}

\subsection{Links}

A link is a disjoint union of circles embedded in $\mathbb{R}^{3}$. A one-component link is called a knot. Links are usually studied via projecting them on a plan; a projection with extra information of overcrossing and undercrossing is called the link diagram.

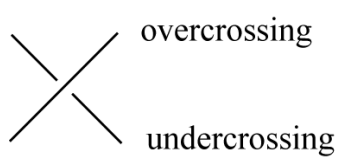

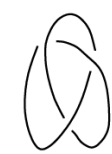

Trefoil knot

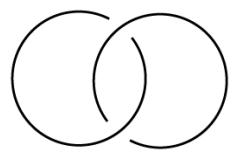

Hopf link

Two links are isotopic if and only if one of them can be transformed to the other by a diffeomorphism of the ambient space onto itself. A fundamental result by Reidemeister [4] about the isotopic link diagrams is: Two unoriented links $L_{1}$ and $L_{2}$ are equivalent if and only if a diagram of $L_{1}$ can be transformed into a diagram of $L_{2}$ by a finite sequence of ambient isotopies of the plane and the local (Reidemeister) moves of the following three types:

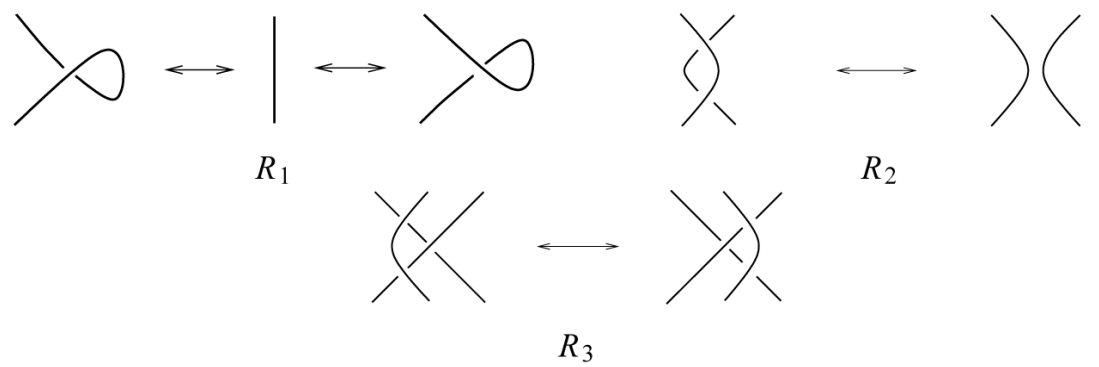

The set of all links that are equivalent to a link $L$ is called a class of $L$. By a link $L$ we shall always mean the class of $L$.

The main question of knot theory is Which two links are equivalent and which are not? To address this question one needs a knot invariant, a function that gives one value on all links that belong to a single class and gives different values (but not always) on knots that belong to different classes. The present work is basically concerned with this question.

\subsection{Braids}

Braids were first studied by Emil Artin in 1925 [5] [6], which now play an important role in knot theory, see [7]-[9] for detail.

An $n$-strand braid is a set of $n$ non intersecting smooth paths connecting $n$ points on a horizontal plane to $n$ points exactly below them on another horizontal plane in an arbitrary order. The smooth paths are called strands of the braid.

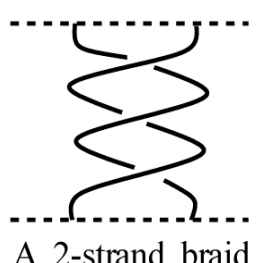

The product $a b$ of two $n$-strand braids is defined by putting the braid $b$ above the braid $a$ and then gluing their common end points.

A braid with only one crossing is called elementary braid. The ith elementary braid $x_{i}$ on $n$ strands is: 


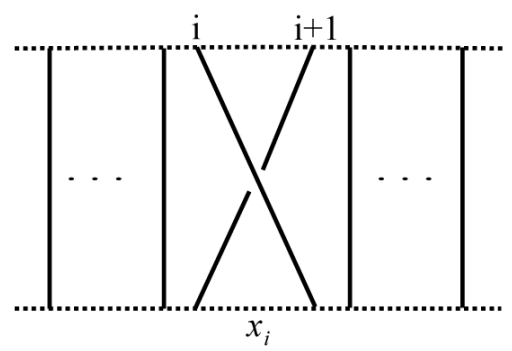

A useful property of elementary braids is that every braid can be written as a product of elementary braids. For instance, the above 2-strand braid is $x_{i}^{-3}=\left(x_{i}^{-1}\right)\left(x_{i}^{-1}\right)\left(x_{i}^{-1}\right)$.

The closure of a braid $b$ is the link $\hat{b}$ obtained by connecting the lower ends of $b$ with the corresponding upper ends.

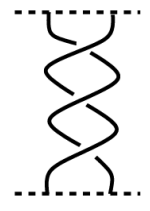

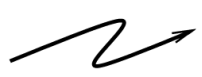

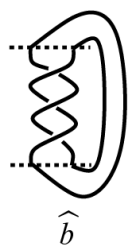

$\widehat{b}$

An important result by Alexander [10] connecting knots and braids is: Each link can be represented as the closure of a braid. This result motivated knot theorists to study braids to solve problems of knot theory.

Remark 2.1 In the last section, all the concerned links will be closures of products of elementary braids.

\subsection{The Kauffman Bracket}

Before the definition it is better to understand the two types of splitting of a crossing, the $A$-type and the $B$-type splittings:

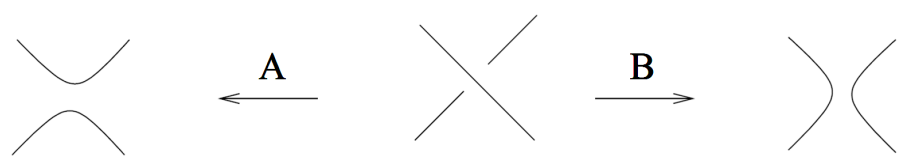

$A$ - and $B$-type splittings

In the following, the symbols $\bigcirc$ and $\sqcup$ represent respectively the unknot and the disconnected sum.

Definition 2.2 The Kauffman bracket is the function $\langle\cdot\rangle:$ Links $\rightarrow \mathbb{Z}\left[a, a^{-1}\right]$ defined by the axioms:

$$
\begin{aligned}
& \langle L\rangle=a\left\langle L_{A}\right\rangle+a^{-1}\left\langle L_{B}\right\rangle \\
& \langle L \sqcup \bigcirc\rangle=\left(-a^{2}-a^{-2}\right)\langle L\rangle \\
& \langle\bigcirc\rangle=1 .
\end{aligned}
$$

Here $L, L_{A}$, and $L_{B}$ are three links which are isotopic everywhere except at one crossing where the look as in the figure:
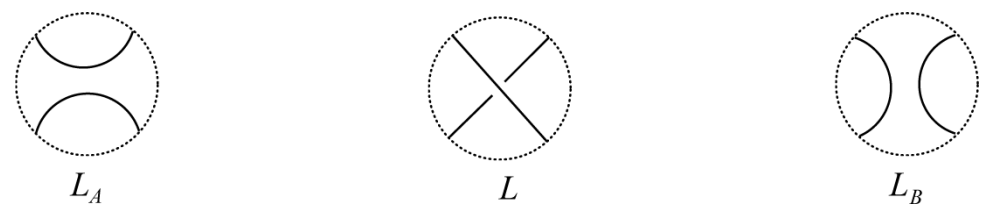

Proposition 2.3 The Kauffman polynomial is invariant under second and third Reidemeister moves but not under the first Reidemeister move [11]. 


\section{Main Results}

In this section we shall introduce a recursive relation for the Kauffman bracket, shall give an explicit formula of $\left\langle\widehat{x_{1}^{n}}\right\rangle$, and shall express $\left\langle\widehat{x_{1}^{m} x_{2}^{n}}\right\rangle$ as the product of $\left\langle\widehat{x_{1}^{m}}\right\rangle$ and $\left\langle\widehat{x_{1}^{n}}\right\rangle$.

First of all we give the Kauffman bracket of the $k$-twist unknot $U_{k}$ :

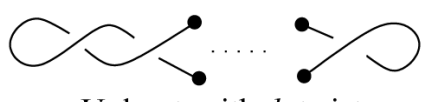

Unknot with $k$ twists

Lemma 3.1 The Kauffman bracket of the $k$-twist unknot is

$$
\left\langle U_{k}\right\rangle=(-1)^{k} a^{3 k} \text {. }
$$

Proof. We prove it by induction on $k$ :

The case $k=0$ holds by definition as $U_{0}$ is the unknot without any crossings. Now, with the assumption that the result holds for an arbitrary $k$, we have

$$
\begin{aligned}
\left\langle U_{k+1}\right\rangle & =\langle\bigcirc\rangle=a\langle\bigcirc\rangle+a^{-1}\langle S\rangle \\
& =a\left(-a^{2}-a^{-2}\right)\left\langle U_{k}\right\rangle+a^{-1}\left\langle U_{k}\right\rangle \\
& =-a^{3}\left\langle U_{k}\right\rangle-a^{3}\left[(-1)^{k} a^{3 k}\right] \\
& =(-1)^{k+1} a^{3(k+1)} .
\end{aligned}
$$

Theorem 3.2 (A recursive relation) The following relation holds for any $n \geq 2$ :

$$
\left\langle\widehat{x_{1}^{n}}\right\rangle=(-1)^{n-1} a^{3 n-2}+a^{-1}\left\langle\widehat{x_{1}^{n-1}}\right\rangle \text {. }
$$

Proof. We prove it using directly the definition and Lemma 3.1:

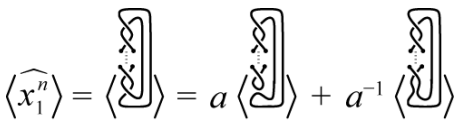

$$
\begin{aligned}
& =a\left\langle U_{n-1}\right\rangle+a^{-1}\left\langle\widehat{x_{1}^{n-1}}\right\rangle \\
& =a\left[(-1)^{n-1} a^{3(n-1)}\right]+a^{-1}\left\langle\widehat{x_{1}^{n-1}}\right\rangle \\
& =(-1)^{n-1} a^{3 n-2}+a^{-1}\left\langle\widehat{x_{1}^{n-1}}\right\rangle .
\end{aligned}
$$

From this recursive relation, we get the explicit formula for the 2-strand braid link $\widehat{x_{1}^{n}}$ :

Proposition 3.3 The Kauffman bracket of the link $\widehat{x_{1}^{n}}, n \geq 2$, is

$$
\left\langle\widehat{x_{1}^{n}}\right\rangle=-a^{-n-2}+\sum_{k=1}^{n-1}(-1)^{n+k-2} a^{(3 n+2)-4 k} .
$$

Proof. We prove it by induction on $n$.

For $n=2$, we have 


$$
\begin{aligned}
& \left\langle\widehat{x_{1}^{2}}\right\rangle=\langle\grave{\Omega}\rangle=a\langle\hat{\Omega}\rangle+a^{-1}\langle\Omega
\end{aligned}
$$

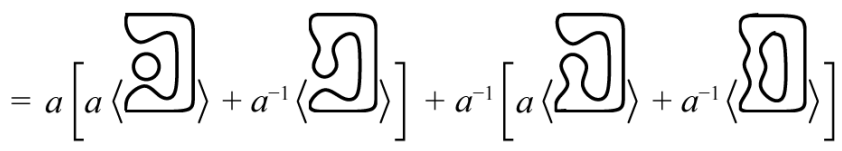

$$
\begin{aligned}
& =a\left[a\left(-a^{2}-a^{-2}\right)+a^{-1}(1)\right]+a^{-1}\left[a(1)+a^{-1}\left(-a^{2}-a^{-2}\right)\right] \\
& =-a^{4}-a^{-4} \text {, }
\end{aligned}
$$

which satisfies the recursive relation.

With the assumption that the relation holds for an arbitrary $n$, we, using Theorem 3.2, get

$$
\begin{aligned}
\left\langle\widehat{x_{1}^{n+1}}\right\rangle= & (-1)^{(n+1)-1} a^{3(n+1)-2}+a^{-1}\left\langle\widehat{x_{1}^{n}}\right\rangle \\
= & (-1)^{n} a^{3(n+1)-2}+a^{-1}\left[(-1)^{n-1} a^{3 n-2}+(-1)^{n-2} a^{3 n-6}\right. \\
& \left.+(-1)^{n-3} a^{3 n-10}+\cdots+(-1)^{2 n-3} a^{-n+6}-a^{-n-2}\right] \\
= & (-1)^{n} a^{3(n+1)-2}+\left[(-1)^{n-1} a^{3 n-3}+(-1)^{n-2} a^{3 n-7}\right. \\
& \left.+(-1)^{n-3} a^{3 n-11}+\cdots+(-1)^{2 n-3} a^{-n+5}-a^{-n-3}\right] \\
= & (-1)^{n} a^{3(n+1)-2}+(-1)^{n-1} a^{3(n+1)-6}+(-1)^{n-2} a^{3(n+1)-10} \\
& +(-1)^{n-3} a^{3(n+1)-14}+\cdots+(-1)^{2(n+1)-3} a^{-(n+1)+6}-a^{-(n+1)-2} .
\end{aligned}
$$

This completes the proof.

In the following we give the Kauffman bracket polynomial of the closure of the braid $\alpha(n)=x_{1} x_{2} x_{1} x_{2} \cdots$ (n factors); this sequence contains the powers of the Garside element $\Delta=x_{1} x_{2} x_{1}=x_{2} x_{1} x_{1}: \alpha(3 k)=\Delta^{k}$.

Proposition 3.4 The Kauffman bracket of $\alpha(n)=x_{1} x_{2} x_{1} \cdots(n$-times) satisfy the recurrence relations:

$$
\begin{aligned}
& \left\langle\Delta^{2 k}\right\rangle=a^{6}\left\langle\Delta^{2(k-1)}\right\rangle-a^{16-6 k}-a^{8-6 k}+a^{4-6 k}+a^{-4-6 k} \\
& \left\langle\Delta^{2 k} x_{1}\right\rangle=a^{6}\left\langle\Delta^{2(k-1)} x_{1}\right\rangle-a^{15-6 k}-a^{7-6 k}+a^{3-6 k}+a^{-5-6 k} \\
& \left\langle\Delta^{2 k} x_{1} x_{2}\right\rangle=a^{6}\left\langle\Delta^{2(k-1)} x_{1} x_{2}\right\rangle-a^{14-6 k}-a^{6-6 k}+a^{2-6 k}+a^{-6-6 k} \\
& \left\langle\Delta^{2 k+1}\right\rangle=a^{6}\left\langle\Delta^{2 k-1}\right\rangle-a^{13-6 k}-a^{5-6 k}+a^{1-6 k}+a^{-7-6 k} \\
& \left\langle\Delta^{2 k+1} x_{2}\right\rangle=a^{6}\left\langle\Delta^{2 k-1} x_{2}\right\rangle-a^{12-6 k}-a^{4-6 k}+a^{-6 k}+a^{-8-6 k} \\
& \left\langle\Delta^{2 k+1} x_{2} x_{1}\right\rangle=a^{6}\left\langle\Delta^{2 k-1} x_{2} x_{1}\right\rangle-a^{11-6 k}-a^{3-6 k}+a^{-1-6 k}+a^{-9-6 k}
\end{aligned}
$$

Proof. Simply, apply the definition for different values of $k$, and write recursively each next bracket in terms of the previous one. $\square$

Lemma 3.5 The Kauffman brackets for $k=0$ are:

$$
\begin{aligned}
& \left\langle\Delta^{0}\right\rangle=a^{4}+2+a^{-4} \\
& \left\langle\Delta^{0} x_{1}\right\rangle=a^{-1}+a^{-5} \\
& \left\langle\Delta^{0} x_{1} x_{2}\right\rangle=a^{-6}
\end{aligned}
$$




$$
\begin{aligned}
& \langle\Delta\rangle=a+a^{-7} \\
& \left\langle\Delta x_{2}\right\rangle=-a^{4}+1+a^{-8} \\
& \left\langle\Delta x_{2} x_{1}\right\rangle=a^{7}-a^{3}+a^{-1}+a^{-9}
\end{aligned}
$$

Proof. The proofs of first three cases are given (proofs of remaining cases are similar):

$$
\begin{aligned}
& \left\langle\Delta^{0}\right\rangle=\langle(0)\rangle=\left(-a^{2}-a^{-2}\right)^{2}\langle\rangle=a^{4}+2+a^{-4} \\
& \left\langle\Delta^{0} x_{1}\right\rangle=\langle\circlearrowright\rangle=\left(-a^{2}-a^{-2}\right)\langle\circlearrowright\rangle=\left(-a^{2}-a^{-2}\right)\left[a\langle\circlearrowright\rangle+a^{-1}\langle\circlearrowright\rangle\right] \\
& =\left(-a^{2}-a^{-2}\right)\left[a+a^{-1}\left(-a^{2}-a^{-2}\right)\right]=a^{-1}+a^{-5} \\
& \left\langle\Delta^{0} x_{1} x_{2}\right\rangle=\langle\sqrt[\Omega]{0}\rangle=a\langle\sqrt[\Omega]{\Omega}\rangle+a^{-1}\langle\sqrt[0]{ }\rangle \\
& =a\left\langle x_{1}\right\rangle+a^{-1}\left(-a^{2}-a^{-2}\right)\left\langle x_{1}\right\rangle=a^{-6}
\end{aligned}
$$

Theorem 3.6 For any $k \geq 0$ the Kauffman bracket of $\alpha(n)=x_{1} x_{2} x_{1} \cdots(n$-times) is given by:

$$
\begin{aligned}
& \left\langle\Delta^{2 k}\right\rangle=2 a^{6 k}+a^{-6 k+4}+a^{-6 k-4} \\
& \left\langle\Delta^{2 k} x_{1}\right\rangle=-a^{6 k+3}+a^{6 k-1}+a^{-6 k+3}+a^{-6 k-5} \\
& \left\langle\Delta^{2 k} x_{1} x_{2}\right\rangle=-a^{6 k+2}+a^{-6 k+2}+a^{-6 k-6} \\
& \left\langle\Delta^{2 k+1}\right\rangle=a^{-6 k+1}+a^{-6 k-7} \\
& \left\langle\Delta^{2 k+1} x_{2}\right\rangle=-a^{6 k+4}+a^{-6 k}+a^{-6 k-8} \\
& \left\langle\Delta^{2 k+1} x_{2} x_{1}\right\rangle=a^{6 k+7}-a^{6 k+3}+a^{-6 k-1}+a^{-6 k-9} .
\end{aligned}
$$

Proof. We prove it by induction on $k$. The case $k=0$ is covered by Lemma 3.5, and the inductive step can be checked with Proposition 3.4.

For instance,

$$
\begin{aligned}
\left\langle\Delta^{2 k+2}\right\rangle & =a^{6}\left\langle\Delta^{2 k}\right\rangle-a^{10-6 k}-a^{2-6 k}+a^{-2-6 k}+a^{-10-6 k} \\
& =a^{6}\left[2 a^{6 k}+a^{4-6 k}+a^{-4-6 k}\right]-a^{10-6 k}-a^{2-6 k}+a^{-2-6 k}+a^{-10-6 k} \\
& =2 a^{6+6 k}+a^{10-6 k}+a^{2-6 k}-a^{10-6 k}-a^{2-6 k}+a^{-10-6 k}+a^{-2-6 k} \\
& =2 a^{6(k+1)}+a^{4-6(k+1)}+a^{-4-6(k+1)} . \quad \square
\end{aligned}
$$

In connected sum $\widehat{x_{1}^{n}} \# U_{k}$ of the braid link $\widehat{x_{1}^{n}}$ with the trivial knot $U_{k}$ has the diagram:

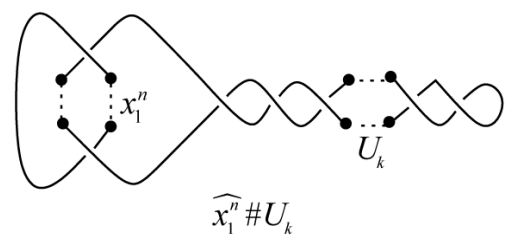

\section{Lemma 3.7}

$$
\left\langle\widehat{x_{1}^{n}} \# U_{k}\right\rangle=(-1)^{k} a^{3 k}\left\langle\widehat{x_{1}^{n}}\right\rangle \text {. }
$$

Proof. We prove it by induction on $k$ : 
For $k=1$, we have

$$
\begin{aligned}
\left\langle\widehat{x_{1}^{n}} \# U_{1}\right\rangle & =\left\langle{ }^{-1}\right\rangle=a\left\langle\widehat{x_{1}^{n}}\right\rangle+a^{-1}\left\langle\widehat{x_{1}^{n}}\right\rangle \\
& =a\left(-a^{2}-a^{-1}\right\rangle \\
& =-a^{3}\left\langle\widehat{x_{1}^{n}}\right\rangle .
\end{aligned}
$$

Now, with the assumption that the result holds for an arbitrary $k$, we have

$$
\begin{aligned}
\left\langle\widehat{x_{1}^{n}} \# U_{k+1}\right\rangle & =a\left\langle\left(-a^{2}-a^{-2}\right)\left\langle\widehat{x_{1}^{n}} \# U_{k}\right\rangle+a^{-1}\left\langle\widehat{x_{1}^{n}} \# U_{k}\right\rangle\right. \\
& =-a^{3}\left\langle\widehat{x_{1}^{n}} \# U_{k}\right\rangle \\
& =-a^{3}\left[(-1)^{k} a^{3 k}\left\langle\widehat{x_{1}^{n}}\right\rangle\right] \\
& =(-1)^{k+1} a^{3(k+1)}\left\langle\widehat{x_{1}^{n}}\right\rangle,
\end{aligned}
$$

as required.

The following result confirms that the Kauffman bracket of $\left\langle\widehat{x_{1}^{m} x_{2}^{n}}\right\rangle$ is actually the product $\left\langle\widehat{x_{1}^{m}}\right\rangle\left\langle\widehat{x_{1}^{n}}\right\rangle$.

Theorem 3.8 For any $m, n \geq 2$,

$$
\left\langle\widehat{x_{1}^{m} x_{2}^{n}}\right\rangle=\left\langle\widehat{x_{1}^{m}}\right\rangle\left\langle\widehat{x_{1}^{n}}\right\rangle
$$

Proof. We prove it by induction on $n$ :

When $n=2$,

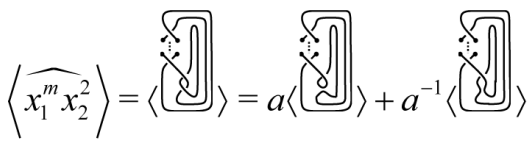

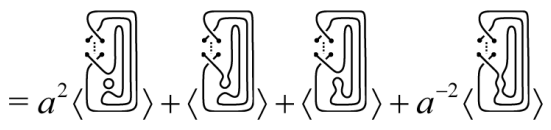

$$
\begin{aligned}
& =a^{2}\left(-a^{2}-a^{-2}\right)\left\langle\widehat{x_{1}^{m}}\right\rangle+\left\langle\widehat{x_{1}^{m}}\right\rangle+\left\langle\widehat{x_{1}^{m}}\right\rangle+a^{-2}\left(-a^{2}-a^{-2}\right)\left\langle\widehat{x_{1}^{m}}\right\rangle \\
& =\left\langle\widehat{x_{1}^{m}}\right\rangle\left[-a^{4}-1+1+1-1-a^{-4}\right] \\
& =\left\langle\widehat{x_{1}^{m}}\right\rangle\left[-a^{4}-a^{-4}\right]=\left\langle\widehat{x_{1}^{m}}\right\rangle\left\langle\widehat{x_{1}^{2}}\right\rangle \text {. }
\end{aligned}
$$

Suppose the result holds for $n=k$, that is $\left\langle\widehat{x_{1}^{m} x_{2}^{k}}\right\rangle=\left\langle\widehat{x_{1}^{m}}\right\rangle\left\langle\widehat{x_{1}^{k}}\right\rangle$.

Now, using Lemma 3.7, we have 


$$
\begin{aligned}
\left\langle\widehat{x_{1}^{m} x_{2}^{k+1}}\right\rangle= & a\left\langle\widehat{x_{1}^{m}} \# U_{k}\right\rangle+a^{-1}\left\langle\widehat{x_{1}^{m} x_{2}^{k}}\right\rangle \\
= & a\left[(-1)^{k} a^{3 k}\left\langle\widehat{x_{1}^{m}}\right\rangle\right]+a^{-1}\left\langle\widehat{x_{1}^{m}}\right\rangle\left\langle\widehat{x_{1}^{k}}\right\rangle \\
= & \left\langle\widehat{x_{1}^{m}}\right\rangle\left[(-1)^{k} a^{3 k+1}+a^{-1}\left\langle\widehat{x_{1}^{k}}\right\rangle\right] \\
= & \left\langle\widehat{x_{1}^{m}}\right\rangle\left[(-1)^{k} a^{3 k+1}+(-1)^{k-1} a^{3 k-3}+(-1)^{k} a^{3 k-7}\right. \\
& \left.+(-1)^{k+1} a^{3 k-11}+\cdots+(-1)^{2 k-2} a^{-k+5}-a^{-k-3}\right] \\
= & \left\langle\widehat{x_{1}^{m}}\right\rangle\left[(-1)^{k-2} a^{3(k+1)-2}+(-1)^{k-1} a^{3(k+1)-6}\right. \\
& \left.+(-1)^{k} a^{3(k+1)-10}+\cdots+(-1)^{2 k-2} a^{-(k+1)+6}-a^{-(k+1)-2}\right] \\
= & \left\langle\widehat{x_{1}^{m}}\right\rangle\left\langle\widehat{x_{1}^{k+1}}\right\rangle .
\end{aligned}
$$

This completes the proof.

\section{Corollary 3.9}

$$
\left\langle\widehat{x_{1}^{m} x_{2}^{n}}\right\rangle=\left\langle\widehat{x_{1}^{n} x_{2}^{m}}\right\rangle \text {. }
$$

Proof. It is obvious: $\left\langle\widehat{x_{1}^{m} x_{2}^{n}}\right\rangle=\left\langle\widehat{x_{1}^{m}}\right\rangle\left\langle\widehat{x_{1}^{n}}\right\rangle=\left\langle\widehat{x_{1}^{n}}\right\rangle\left\langle\widehat{x_{1}^{m}}\right\rangle=\left\langle\widehat{x_{1}^{n} x_{2}^{m}}\right\rangle$.

\section{Corollary 3.10}

$$
\operatorname{deg}\left\langle\widehat{x_{1}^{m} x_{2}^{n}}\right\rangle=3(m+n)-4
$$

and

$$
\operatorname{span}\left\langle\widehat{x_{1}^{m} x_{2}^{n}}\right\rangle=4(m+n)
$$

Proof. The result follows immediately from Theorem 3.8 as $\operatorname{deg}\left\langle\widehat{x_{1}^{n}}\right\rangle=3 n-2$ and span $\left\langle\widehat{x_{1}^{n}}\right\rangle=-n-2$.

For the following, let us fix the notation $L_{a b c}$ for the link with the understanding that the link contains $a, b$, and $c$ crossings of type $x_{1}, x_{2}$, and $x_{1}$, respectively, and that $L_{a b c} \neq \widehat{x_{1}^{a} x_{2}^{b}} x_{1}^{a}$.

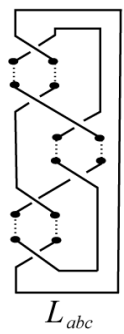

Proposition 3.11 The Kauffman bracket of the link $L_{a b c}$ is

$$
\left\langle L_{a b c}\right\rangle=\left\langle\widehat{x_{1}^{a}}\right\rangle\left\langle\widehat{x_{1}^{b}}\right\rangle\left\langle\widehat{x_{1}^{c}}\right\rangle+\left(a^{-b+2}+a^{-b-2}\right)\left\langle\widehat{x_{1}^{a}}\right\rangle\left\langle\widehat{x_{1}^{c}}\right\rangle+a^{-b}\left\langle\widehat{x_{1}^{a+c}}\right\rangle
$$

Proof. We prove it by induction on $b$ :

For $b=1$, we have 


$$
\begin{aligned}
\left\langle L_{a 1 c}\right\rangle & =\left\langle\widehat{x_{1}^{a}}\right\rangle\left\langle\widehat{x_{1}^{1}}\right\rangle\left\langle\widehat{x_{1}^{c}}\right\rangle+\left(a+a^{-3}\right)\left\langle\widehat{x_{1}^{a}}\right\rangle\left\langle\widehat{x_{1}^{c}}\right\rangle+a^{-1}\left\langle\widehat{x_{1}^{a+c}}\right\rangle \\
& =a^{-3}\left\langle\widehat{x_{1}^{a}}\right\rangle\left\langle\widehat{x_{1}^{c}}\right\rangle+\left(a+a^{-3}\right)\left\langle\widehat{x_{1}^{a}}\right\rangle\left\langle\widehat{x_{1}^{c}}\right\rangle+a^{-1}\left\langle\widehat{x_{1}^{a+c}}\right\rangle \\
& =a\left\langle\widehat{x_{1}^{a}}\right\rangle\left\langle\widehat{x_{1}^{c}}\right\rangle+a^{-1}\left\langle\widehat{x_{1}^{a+c}}\right\rangle .
\end{aligned}
$$

Now, with the assumption that the result holds for an arbitrary $k$, we have $b=k+1$

$$
\begin{aligned}
\left\langle L_{a(k+1) c}\right\rangle= & a\left\langle\widehat{x_{1}}\right\rangle+a_{k}^{-1}\left\langle\widehat{x_{1}^{c}}\right\rangle+a^{-1}\left\langle\widehat{x_{1}^{a} x_{2}^{k} x_{1}^{c}}\right\rangle \\
= & a\left\langle\widehat{x^{a}}\right. \\
= & a\left\langle\widehat{x_{1}^{a}} \# U_{k}\right\rangle\left\langle\widehat{x_{1}^{c}}\right\rangle+a^{-1}\left\langle\widehat{x_{1}^{a} x_{2}^{k} x_{1}^{c}}\right\rangle \\
= & a(-1)^{k} a^{3 k}\left\langle\widehat{x_{1}^{a}}\right\rangle\left\langle\widehat{x_{1}^{c}}\right\rangle+a^{-1}\left(\left\langle\widehat{x_{1}^{a}}\right\rangle\left\langle\widehat{x_{1}^{k}}\right\rangle\left\langle\widehat{x_{1}^{c}}\right\rangle+\left(a^{-k+2}+a^{-k-2}\right)\left\langle\widehat{x_{1}^{a}}\right\rangle\left\langle\widehat{x_{1}^{c}}\right\rangle+a^{-k}\left\langle\widehat{x_{1}^{a+c}}\right\rangle\right) \\
= & (-1)^{k} a^{3 k+1}\left\langle\widehat{x_{1}^{a}}\right\rangle\left\langle\widehat{x_{1}^{c}}\right\rangle+a^{-1}\left\langle\widehat{x_{1}^{a}}\right\rangle\left\langle\widehat{x_{1}^{k}}\right\rangle\left\langle\widehat{x_{1}^{c}}\right\rangle+\left(a^{-k+1}+a^{-k-3}\right)\left\langle\widehat{x_{1}^{a}}\right\rangle\left\langle\widehat{x_{1}^{c}}\right\rangle+a^{-k-1}\left\langle\widehat{x_{1}^{a+c}}\right\rangle \\
= & \left((-1)^{k} a^{3 k+1}+(-1)^{k+1} a^{3 k-3}+\cdots-a^{-k+5}-a^{-k-3}\right)\left\langle\widehat{x_{1}^{a}}\right\rangle\left\langle\widehat{x_{1}^{c}}\right\rangle \\
& +\left(a^{-k-1+2}+a^{-k-1-2}\right)\left\langle\widehat{x_{1}^{a}}\right\rangle\left\langle\widehat{x_{1}^{c}}\right\rangle+a^{-k-1}\left\langle\widehat{x_{1}^{a+c}}\right\rangle \\
= & \left\langle\widehat{x_{1}^{a}}\right\rangle\left\langle\widehat{x_{1}^{k+1}}\right\rangle\left\langle\widehat{x_{1}^{c}}\right\rangle+\left(a^{-(k+1)+2}+a^{-(k+1)-2}\right)\left\langle\widehat{x_{1}^{a}}\right\rangle\left\langle\widehat{x_{1}^{c}}\right\rangle+a^{-(k+1)}\left\langle\widehat{x_{1}^{a+c}}\right\rangle
\end{aligned}
$$

as required. $\square$

Proposition 3.12 The Kauffman bracket of the link $\overline{x_{1}^{a} x_{2}^{b} x_{1}^{c} x_{2}^{d}}$ is

$$
\begin{aligned}
\left\langle\widehat{x_{1}^{a} x_{2}^{b} x_{1}^{c} x_{2}^{d}}\right\rangle= & \left(\sum_{i=1}^{d}(-1)^{d+i} a^{3 d-4 i+2}\right)\left\langle\widehat{x_{1}^{a}}\right\rangle\left\langle\widehat{x_{1}^{b}}\right\rangle\left\langle\widehat{x_{1}^{c}}\right\rangle \\
& +\left((-1)^{d+1} a^{-b+3 d}+a^{-b-d}\right)\left\langle\widehat{x_{1}^{a}}\right\rangle\left\langle\widehat{x_{1}^{c}}\right\rangle \\
& +\left(\sum_{i=1}^{d}(-1)^{d+i} a^{3 d-4 i+2-b}+a^{-d}\left\langle\widehat{x_{1}^{b}}\right\rangle\right)\left\langle\widehat{x_{1}^{a+c}}\right\rangle .
\end{aligned}
$$

Proof. We prove it by induction on $d$ :

For $d=1$, we have

$$
\begin{aligned}
\left\langle\widehat{x_{1}^{a} x_{2}^{b} x_{1}^{c} x_{2}^{1}}\right\rangle= & \left(\sum_{i=1}^{1}(-1)^{d+i} a^{3-4 i+2}\right)\left\langle\widehat{x_{1}^{a}}\right\rangle\left\langle\widehat{x_{1}^{b}}\right\rangle\left\langle\widehat{x_{1}^{c}}\right\rangle+\left((-1)^{d+1} a^{-b+3}+a^{-b-1}\right)\left\langle\widehat{x_{1}^{a}}\right\rangle\left\langle\widehat{x_{1}^{c}}\right\rangle \\
& +\left(\sum_{i=1}^{1}(-1)^{1+i} a^{3-4 i+2-b}+a^{-1}\left\langle\widehat{x_{1}^{b}}\right\rangle\right)\left\langle\widehat{x_{1}^{a+c}}\right\rangle \\
= & a\left\langle\widehat{x_{1}^{a}}\right\rangle\left\langle\widehat{x_{1}^{b}}\right\rangle\left\langle\widehat{x_{1}^{c}}\right\rangle+\left(a^{-b+3}+a^{-b-1}\right)\left\langle\widehat{x_{1}^{a}}\right\rangle\left\langle\widehat{x_{1}^{c}}\right\rangle \\
& +\left(a^{-b+1}+a^{-1}\left\langle\widehat{x_{1}^{b}}\right\rangle\right)\left\langle\widehat{x_{1}^{a+c}}\right\rangle .
\end{aligned}
$$

Now, with the assumption that the result holds for $d=k$, we have 


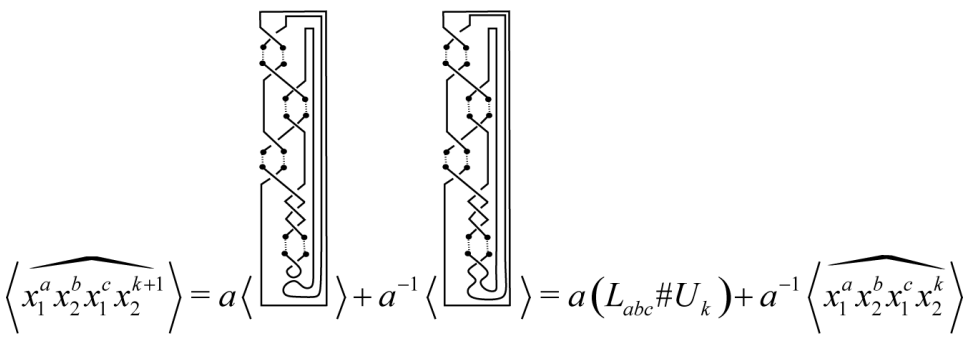

$$
\begin{aligned}
& =a\left[(-1)^{k} a^{3 k}\left(\left\langle\widehat{x_{1}^{a}}\right\rangle\left\langle\widehat{x_{1}^{b}}\right\rangle\left\langle\widehat{x_{1}^{c}}\right\rangle+\left(a^{-b+2}+a^{-b-2}\right)\left\langle\widehat{x_{1}^{a}}\right\rangle\left\langle\widehat{x_{1}^{c}}\right\rangle+a^{-b}\left\langle\widehat{x_{1}^{a+c}}\right\rangle\right)\right] \\
& +a^{-1}\left[\left(\sum_{i=1}^{k}(-1)^{k+i} a^{3 k-4 i+2}\right)\left\langle\widehat{x_{1}^{a}}\right\rangle\left\langle\widehat{x_{1}^{b}}\right\rangle\left\langle\widehat{x_{1}^{c}}\right\rangle+\left((-1)^{k+1} a^{-b+3 k}+a^{-b-k}\right)\left\langle\widehat{x_{1}^{a}}\right\rangle\left\langle\widehat{x_{1}^{c}}\right\rangle\right. \\
& \left.+\left(\sum_{i=1}^{k}(-1)^{k+i} a^{3 k-4 i+2-b}+a^{-k}\left\langle\widehat{x_{1}^{b}}\right\rangle\right)\left\langle\widehat{x_{1}^{a+c}}\right\rangle\right] \\
& =(-1)^{k} a^{3 k+1}\left\langle\widehat{x_{1}^{a}}\right\rangle\left\langle\widehat{x_{1}^{b}}\right\rangle\left\langle\widehat{x_{1}^{c}}\right\rangle+\left((-1)^{k} a^{3 k+3-b}+(-1)^{k} a^{3 k-1-b}\right)\left\langle\widehat{x_{1}^{a}}\right\rangle\left\langle\widehat{x_{1}^{c}}\right\rangle \\
& +(-1)^{k} a^{3 k+1-b}+\sum_{i=1}^{k}(-1)^{k+i} a^{3 k-4 i+1}\left\langle\widehat{x_{1}^{a}}\right\rangle\left\langle\widehat{x_{1}^{b}}\right\rangle\left\langle\widehat{x_{1}^{c}}\right\rangle \\
& +\left((-1)^{k+1} a^{3 k-1-b}+a^{-k-1-b}\right)\left\langle\widehat{x_{1}^{a}}\right\rangle\left\langle\widehat{x_{1}^{c}}\right\rangle+\left(\sum_{i=1}^{k}(-1)^{k+i} a^{3 k-4 i+1-b}+a^{-k-1}\left\langle\widehat{x_{1}^{b}}\right\rangle\right)\left\langle\widehat{x_{1}^{a+c}}\right\rangle \\
& =\left(\sum_{i=1}^{k+1}(-1)^{(k+1)+i} a^{3(k+1)-4 i+2}\right)\left\langle\widehat{x_{1}^{a}}\right\rangle\left\langle\widehat{x_{1}^{b}}\right\rangle\left\langle\widehat{x_{1}^{c}}\right\rangle+\left((-1)^{(k+1)+1} a^{-b+3(k+1)}+a^{-b-(k+1)}\right)\left\langle\widehat{x_{1}^{a}}\right\rangle\left\langle\widehat{x_{1}^{c}}\right\rangle \\
& +\left(\sum_{i=1}^{k+1}(-1)^{(k+1)+i} a^{3(k+1)-4 i+2-b}+a^{-(k+1)}\left\langle\widehat{x_{1}^{b}}\right\rangle\right)\left\langle\widehat{x_{1}^{a+c}}\right\rangle,
\end{aligned}
$$

as was required.

\section{References}

[1] Kauffman, L.H. (1987) State Models and the Jones Polynomial. Topology, 26, 395-407. http://dx.doi.org/10.1016/0040-9383(87)90009-7

[2] Jaeger, F. (1990) A Combinatorial Model for the Homy Polynomial. European Journal of Combinatorics, 11, 549-555.

[3] Reshetikhin, N.Y. (1988) Quantized Universal Enveloping Algebras, the Yang-Baxter Equation and Invariants of Links, I and II. LOMI Reprints E-4-87 and E-17-87, Steklov Institute, Leningrad, USSR.

[4] Reidemeister, K. (1948) Knot Theory. Chelsea Publ and Co., New York.

[5] Artin, E. (1925) Theorie der Z Ö pfe. Abhandlungen aus dem Mathematischen Seminar der Universität Hamburg, 4, 27-72. http://dx.doi.org/10.1007/BF02950718

[6] Artin, E. (1947) Theory of Braids. Annals of Mathematics, 48, 101-126. http://dx.doi.org/10.2307/1969218

[7] Birman, J.S. (1974) Braids, Links, and Mapping Class Groups. Princeton University Press, Princeton.

[8] Manturov, V.O. (2004) Knot Theory. Chapman and Hall/CRC, Boca Raton. http://dx.doi.org/10.1201/9780203402849

[9] Murasugi, K. (1996) Knot Theory and Its Applications. Birkh ä User, Boston.

[10] Alexander, J. (1923) Topological Invariants of Knots and Links. Transactions of the American Mathematical Society, 20, 275-306.

[11] Adams, C.C. (1994) The Knot Book. W H Freeman and Company, New York. 
Scientific Research Publishing (SCIRP) is one of the largest Open Access journal publishers. It is currently publishing more than 200 open access, online, peer-reviewed journals covering a wide range of academic disciplines. SCIRP serves the worldwide academic communities and contributes to the progress and application of science with its publication.

Other selected journals from SCIRP are listed as below. Submit your manuscript to us via either submit@scirp.org or Online Submission Portal.
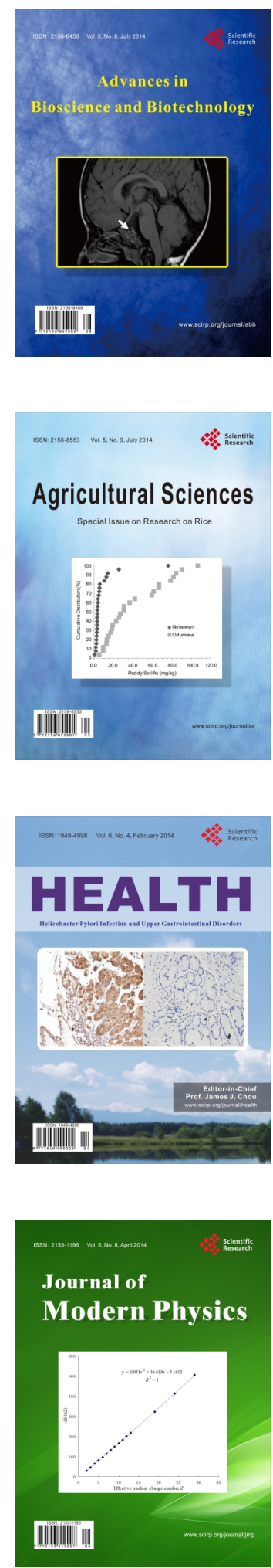
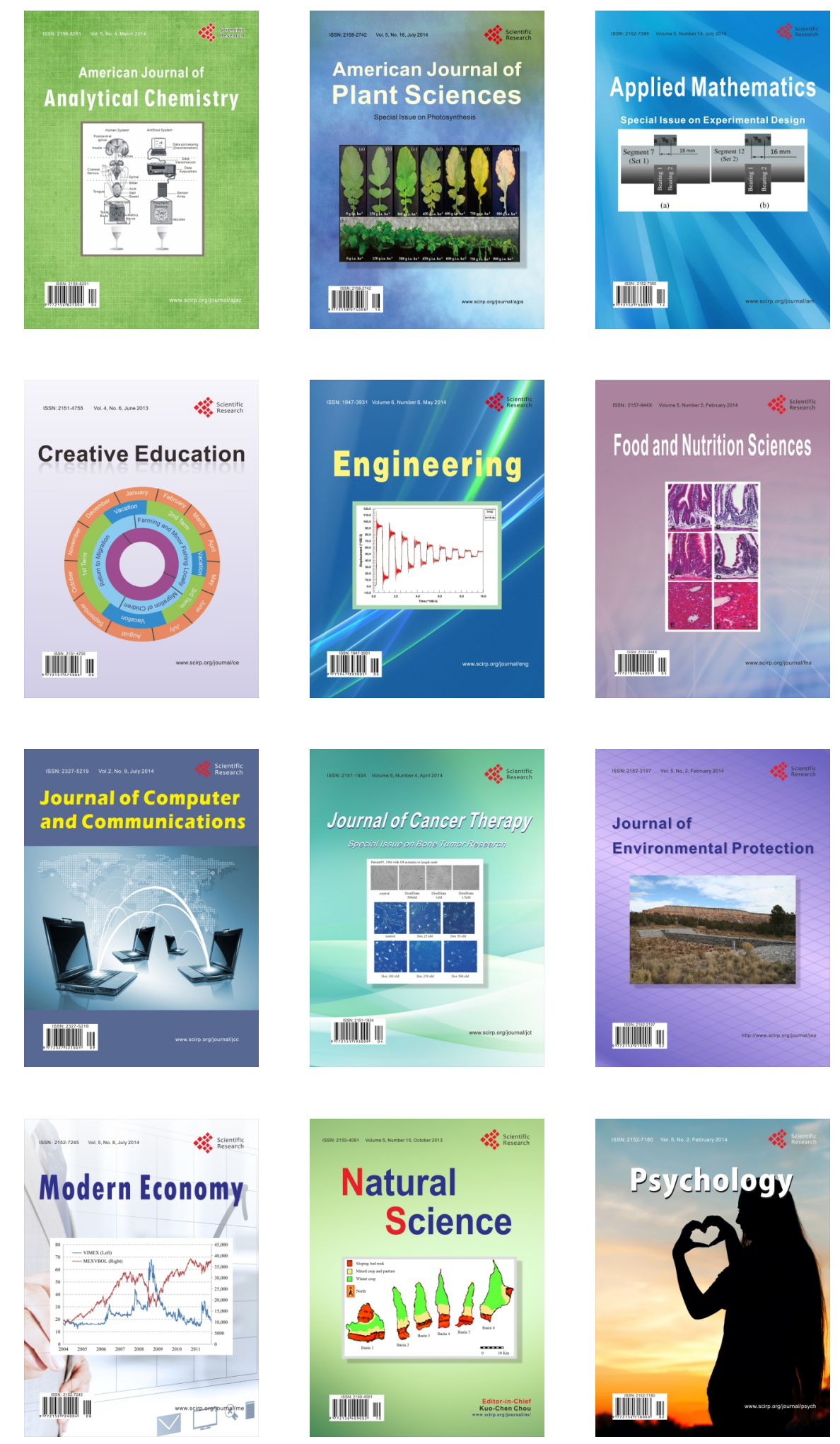